\title{
Manipulation of two regulatory genes for efficient production of chromomycins in Streptomyces reseiscleroticus
}

\author{
Lei Sun ${ }^{1}$, Jia Zeng ${ }^{1}$, Peiwu Cui ${ }^{2}$, Wei Wang ${ }^{2}$, Dayu $\mathrm{Yu}^{3}$ and Jixun Zhan ${ }^{1,2^{*}}$
}

\begin{abstract}
Background: Regulatory genes play critical roles in natural product biosynthetic pathways. Chromomycins are promising anticancer natural products from actinomycetes. This study is aimed to create an efficient strain for production of these molecules by manipulating the regulatory genes.
\end{abstract}

Results: A putative but silent chromomycin biosynthetic gene cluster was discovered in Streptomyces reseiscleroticus. Heterologous expression of the ketosynthase, chain length factor, and acyl carrier protein in Streptomyces lividans confirmed that they are responsible for the assembly of a decaketide. Two regulatory genes are present in this gene cluster, including SARP-type activator SrcmRI and PadR-like repressor SrcmRll. Either overexpression of SrcmRI or disruption of SrcmRll turned on the biosynthetic pathway of chromomycins. The production titers of chromomycin $A_{3} / A_{2}$ in $R 5$ agar in these two strains reached $8.9 \pm 1.2 / 13.2 \pm 1.6$ and $49.3 \pm 4.3 / 53.3 \pm 3.6 \mathrm{mg} / \mathrm{L}$, respectively. An engineered strain was then constructed with both SrcmRll disruption and SrcmRl overexpression, which produced chromomycins $A_{3}$ and $A_{2}$ in $R 5$ agar at $69.4 \pm 7.6$ and $81.7 \pm 7.2 \mathrm{mg} / \mathrm{L}$, respectively. Optimization of the culture conditions further increased the titers of chromomycins $A_{3}$ and $A_{2}$ respectively to $145.1 \pm 15.3$ and $158.3 \pm 15.4 \mathrm{mg} / \mathrm{L}$ in liquid fermentation.

Conclusions: This work revealed the synergistic effect of manipulation of pathway repressor and activator genes in the engineering of a natural product biosynthetic pathway. The resulting engineered strain showed the highest production titers of chromomycins by a strain of Streptomyces, providing an efficient way to produce these pharmaceutically valuable molecules.

Keywords: Streptomyces reseiscleroticus, Chromomycins, Biosynthesis, PadR-like transcription regulator, SARP regulator

\section{Background}

Chromomycins are glycosylated polyketide compounds that were first found in Streptomyces griseus. These natural products belong to a group of cancerostatic and antitumor antibiotics [1]. Chromomycins and mithramycins, representative compounds of the aureolic acid family, are effective against various human tumors such as brain and testicular carcinomas [2, 3]. Chromomycin $\mathrm{A}_{3}$ (1, Fig. 1) was also reported to enable the stimulation of $\mathrm{K} 562$ cell erythroid

\footnotetext{
* Correspondence: jixun.zhan@usu.edu

1Department of Biological Engineering, Utah State University, 4105 Old Main Hill, Logan, UT 84322-4105, USA

${ }^{2} \mathrm{TCM}$ and Ethnomedicine Innovation \& Development Laboratory, School of Pharmacy, Hunan University of Chinese Medicine, Changsha, Hunan 410208, China

Full list of author information is available at the end of the article
}

differentiation [4]. It was used in the neurological therapeutics [5] and showed activities against HIV-1 [6]. 1 has been clinically used as an anticancer drug since 1960s [7]. Chromomycin $\mathrm{A}_{2}$ (2, Fig. 1) is a structurally similar analog of $\mathbf{1}$ and has shown similar bioactivities.

Antibiotic biosynthesis is typically controlled at different levels. The most fundamental level involves pleiotropic regulators which control both secondary metabolism and morphological differentiation. At the next level, regulatory genes exert their control over two or more antibiotic biosynthetic pathways in the same organism, but have no effects on morphological differentiation. Finally, the most specific level of control concerns genes encoding regulators that act only on one biosynthetic pathway [8]. These genes, whose expression is partly dependent on pleiotropic regulators, are usually found physically linked to

(c) The Author(s). 2018 Open Access This article is distributed under the terms of the Creative Commons Attribution 4.0 International License (http://creativecommons.org/licenses/by/4.0/), which permits unrestricted use, distribution, and 


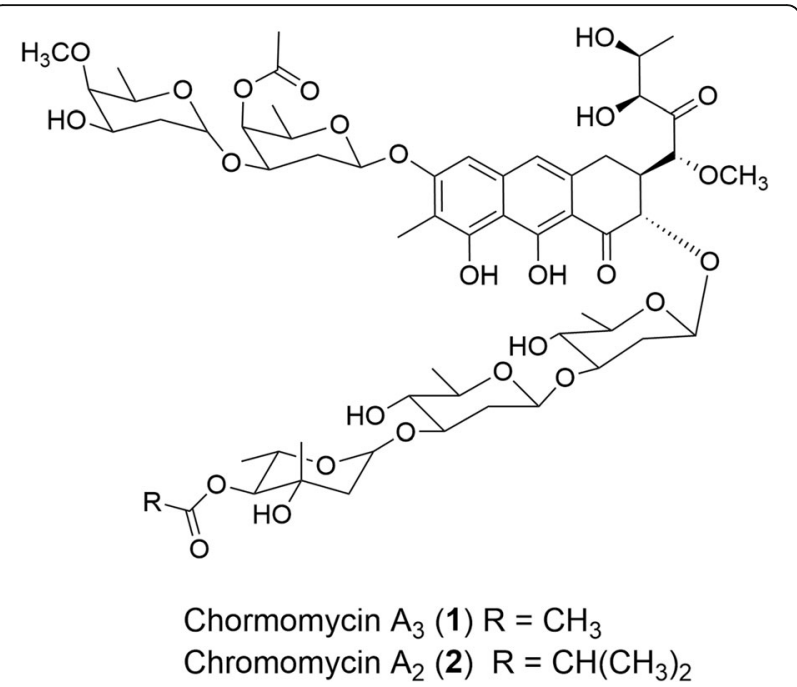

Fig. 1 Structures of chromomycin $A_{3}(\mathbf{1})$ and $A_{2}(\mathbf{2})$

the structural antibiotic biosynthetic genes on the chromosomes of streptomycetes. This is different from the global regulatory genes, which can be located far from the clusters they control. In most cases, the pathway-specific regulators play a positive role in the transcription of the biosynthetic genes, while others may act as transcriptional repressors. The group of proteins referred to as Streptomyces antibiotic regulatory proteins (SARPs) is prominent among the pathway-specific regulators in streptomycetes [8].

In Gram-positive bacteria, the phenolic acid decarboxylase $(\mathrm{Pad})$ gene is involved in the phenolic acid stress response. Phenolic acids are decarboxylated by inducible Pad enzymes into vinyl phenol derivatives, which are not toxic to Gram-positive bacteria. The phenolic acid decarboxylase repressor (PadR) gene encodes the transcriptional regulator that represses genes associated with the phenolic acid stress response in Gram-positive bacteria such as Bacillus subtilis, Pediococcus pentosaceus, and Lactobacillus plantarum [9-11]. Among these transcriptional regulators, only a few of the PadR-like proteins have been investigated, such as AphA which activates virulence gene expression in Vibrio cholera [12], LmrR and LadR that inhibit the expression of a multi-drug resistance pump in Lactococcus lactis and Listeria monocytogenesis, respectively [13, 14], and CmmRII, a transcriptional repressor of chromomycin resistance/biosynthesis in S. griseus subsp. griseus [15].

S. roseiscleroticus (ATCC ${ }^{\circ}$ 53903) was known to produce an antitumor drug, sultriecin [16]. The genome of this strain has been recently sequenced by our group. One putative type II polyketide synthase (PKS) gene cluster was discovered in this strain, which could be involved in the biosynthesis of chromomycins based on bioinformatics analysis. We also found two regulatory genes in the gene cluster, including the activator ( $\mathrm{srcmRII})$ and repressor $(\mathrm{srcmRI})$ genes, which may play roles in the regulation of the whole gene cluster. By manipulating these two regulatory genes, we were able to turn on the silent chromomycin biosynthetic gene cluster, and create efficient producing strains. The production process was optimized and the titers of $\mathbf{1}$ and $\mathbf{2}$ reached $145.1 \pm 15.3$ and $158.3 \pm 15.4 \mathrm{mg} / \mathrm{L}$, respectively.

\section{Results}

Analysis of the putative chromomycin biosynthetic gene cluster

The genomic DNA of Streptomyces reseiscleroticus was sequenced by our group. Annotation of the genes revealed a type II polyketide biosynthetic gene cluster that consists of 36 genes and might be involved in the biosynthesis of $\mathbf{1}$ and 2. Mithramycin shares the same aglycon structure with chromomycins, and its biosynthetic pathway has been well studied [17]. The putative chromomycin ( $\mathrm{srcm}$ ) biosynthetic gene cluster in $S$. reseiscleroticus is similar to that for mithramycin [18], and almost identical to that for chromomycin $\mathrm{A}_{3}$ in $S$. griseus (Fig. 2a) [1]. Three enzymes SrcmP, K and $\mathrm{S}$ are the minimal PKS, which might be responsible for the biosynthesis of the decaketide backbone of the chromomycin aglycon. SrcmQ, X and Y would be involved in cyclization and aromatization process. SrcmOI, OII and OIV are predicted to be oxygenases involved in the hydroxylations of aromatic polyketides. Since the chromomycin aglycon contains two methyl groups, SrcmMI and MII are proposed to be involved in these methylation steps. There are several enzymes predicted to be involved in the formation and transfer of the deoxysugar moieties, such as SrcmD, E, F, V, WI, and MIII, the glycosyltransferases SrcmGI-IV, and NDP-hexose 4-ketoreductase SrcmUI-III. The biosynthetic pathway of 1 and $\mathbf{2}$ was proposed in Fig. 2b. SrcmH, I, and J might be involved in self-resistance. SrcmRI and RII are putative regulatory proteins, likely involved in the regulation of the biosynthetic pathway of chromomycins (Table 1).

\section{Heterologous expression of the type II minimal PKS in S. lividans K4-114}

According to the annotation of the gene cluster in $S$. roseiscleroticus, srcmPKS are putative minimal PKS genes in chromomycin biosynthesis. In order to confirm the function of SrcmPKS, we constructed a plasmid pRM5-srcmPKS (pSUN6) and introduced it into S. lividans K4-114 for heterologous expression. As shown in trace $\mathrm{i}$ of Fig. 3a, a major product 3 at $21.6 \mathrm{~min}$ was observed in the extract of engineered strain KS1. For the reference, we constructed another pRM5-based expression plasmid (pTZ3) that carries the OxyABC genes 


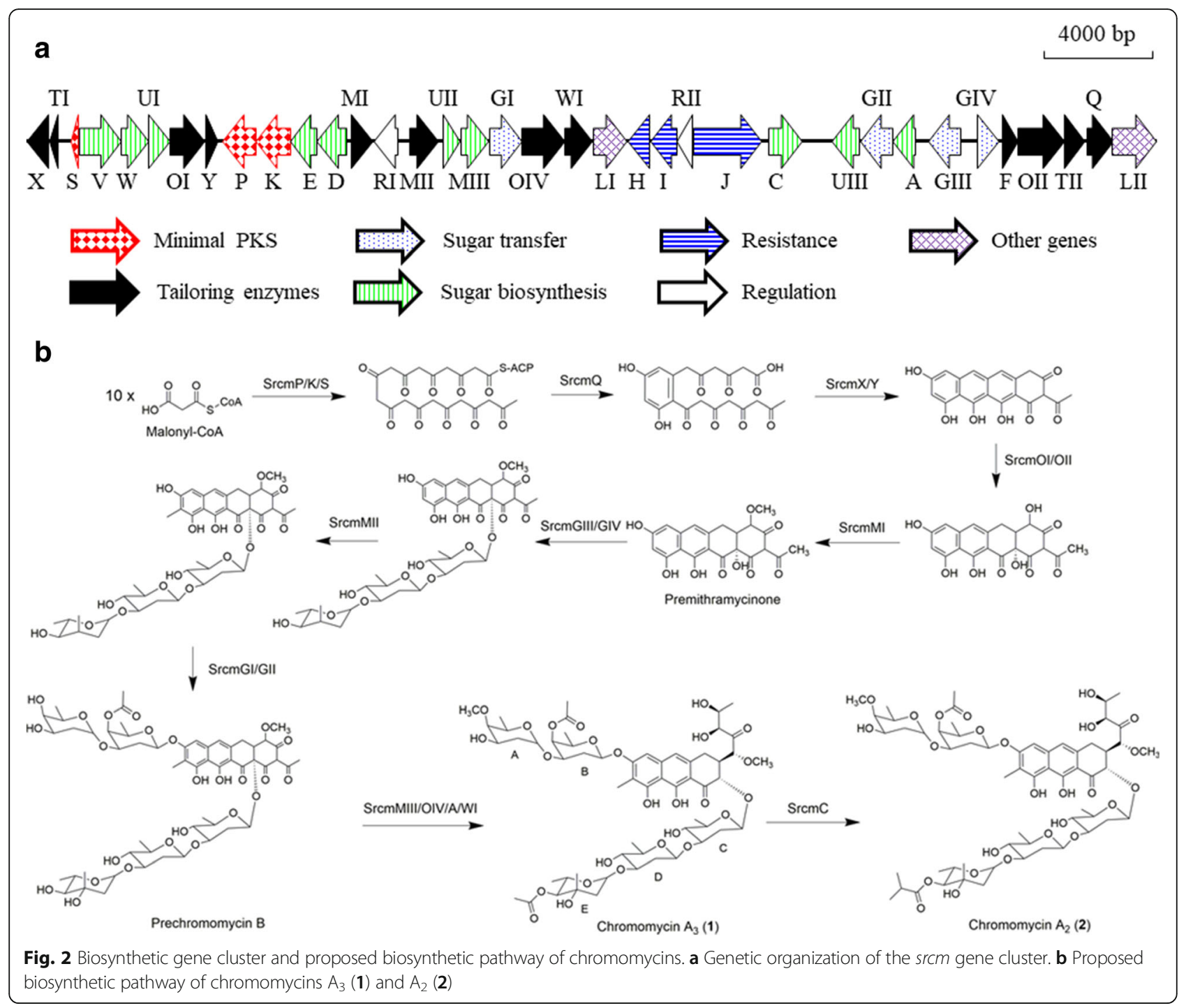

encoding the oxytetracycline minimal PKS in Streptomyces rimosus. As shown in trace ii of Fig. 3a, a peak was eluted at the same time as 3, whereas S. lividans K4-114 harboring the empty vector pRM5 did not produce any polyketide products (trace iii, Fig. 3a). The oxytetracycline minimal PKS OxyABC was previously reported to synthesize a decaketide which will be spontaneously cyclized into SEK15b (Fig. 3b) [19]. These results indicated that SrcmPKS has the same function as OxyABC.

In order to isolate and identify compound 3, a scaled up culture of S. lividans K4-114/pSUN6 was prepared. ESI-MS analysis of 3 showed ion peaks $[\mathrm{M}+\mathrm{H}]^{+}$at $\mathrm{m} / z$ 381.0 and $[\mathrm{M}-\mathrm{H}]^{-} \mathrm{m} / z$ 379.1 (Additional file 1: Figure S1a), indicating that the molecular weight of this compound is 380, which corresponds to that of SEK15b. The ${ }^{1}$ H NMR (Additional file 1: Table S1) of 3 indicated that the compound has five aromatic proton signals and one high field methyl group signal. Twenty carbons were observed in the ${ }^{13} \mathrm{C}$ NMR spectrum (Additional file 1: Table S1) of 3. After a comparison with the published data [20], compound 3 was identified as SEK15b. Therefore, SrcmPKS were confirmed to be the minimal PKS for the biosynthesis of a decaketide.

\section{Disruption of srcmRII in S. roseiscleroticus and products characterization}

After we confirmed that the decaketide was synthesized by SrcmPKS, we sought to search for chromomycins in the extract of wild type S. roseiscleroticus. However, there were no target peaks observed (trace i, Fig. 4a). Thus, this gene cluster was considered silent in S. roseiscleroticus under the lab conditions.

Regulatory genes play important roles in controlling the expression of antibiotic biosynthetic pathways. srcmRII is one of the regulatory genes in the chromomycin biosynthetic gene cluster. BLAST analysis indicated 
Table 1 Putative functions of the genes in the chromomycin biosynthetic gene cluster

\begin{tabular}{|c|c|c|c|c|}
\hline Gene & Annotation & Size (aa) & Homologue & Identities \\
\hline $\operatorname{srcm} Y$ & cyclase & 258 & DacN [Dactylosporangium sp. SC14051] & $70 \%$ \\
\hline srcmTl & ketoreductase & 258 & CmmTI [Streptomyces griseus subsp. griseus] & $64 \%$ \\
\hline srems & acyl carrier protein & 85 & Acyl carrier protein [Streptomyces sp. 275] & $100 \%$ \\
\hline sremV & NDP-hexose 2,3-dehydratase & 462 & PnxS3 [Streptomyces sp. TA-0256] & $54 \%$ \\
\hline $\operatorname{srcmW}$ & NDP-hexose 3-ketoreductase & 331 & PokS4 [Streptomyces diastatochromogenes] & $53 \%$ \\
\hline srcmUII & NDP-hexose 4-ketoreductase & 252 & SaqR [Micromonospora sp. Tu 6368] & $53 \%$ \\
\hline srcmOl & FAD-dependent monooxyngease & 423 & CmmOI [Streptomyces griseus subsp. griseus] & $70 \%$ \\
\hline $\operatorname{srcm} X$ & cyclase & 142 & DacG [Dactylosporangium sp. SC14051] & $60 \%$ \\
\hline srcmk & chain length factor & 402 & SaqB [Micromonospora sp. Tu 6368] & $66 \%$ \\
\hline $\mathrm{srcm} P$ & ketosynthase & 422 & SaqA [Micromonospora sp. Tu 6368] & $76 \%$ \\
\hline srcmE & dTDP-glucose 4,6-dehydratase & 326 & CmmE [Streptomyces griseus subsp. griseus] & $75 \%$ \\
\hline srcmD & NDP-glucose synthase & 355 & Lct49 [Streptomyces rishiriensis] & $57 \%$ \\
\hline srcmMIII & sugar O-methyltransferase & 252 & AlmCll [Streptomyces sp. A1] & $61 \%$ \\
\hline srcmRl & SARP family transcriptional regulator & 292 & CmmRI [Streptomyces griseus subsp. griseus] & $71 \%$ \\
\hline srcmMIl & C-methyltransferase & 343 & mtmMII [Streptomyces argillaceus] & $57 \%$ \\
\hline srcmUl & NDP-hexose 4-ketoreductase & 254 & ChaR [Streptomyces chattanoogensis] & $54 \%$ \\
\hline sremMl & O-methyltransferase & 344 & CtcO [Kitasatospora aureofaciens] & $51 \%$ \\
\hline srcmGl & glycosyltransferase & 394 & PyrC4 [Streptomyces rugosporus] & $41 \%$ \\
\hline srcmOIV & FAD-dependent monooxygenase & 511 & mtmOIV [Streptomyces argillaceus] & $55 \%$ \\
\hline srcmWl & side-chain ketoreductase & 326 & MtmW [Streptomyces argillaceus] & $64 \%$ \\
\hline $\operatorname{srcmLl}$ & acyl CoA ligase & 409 & CmmLI [Streptomyces griseus subsp. griseus] & $68 \%$ \\
\hline $\mathrm{srcmH}$ & $A B C$ transporter membrane protein & 251 & SfrB [Streptomyces sp. 275] & $100 \%$ \\
\hline $\mathrm{srcml}$ & ABC transporter ATP-binding protein & 322 & SfrA [Streptomyces sp. 275] & $100 \%$ \\
\hline sremRll & PadR family transcriptional regulator & 186 & CmmRII [Streptomyces griseus subsp. griseus] & $77 \%$ \\
\hline $\mathrm{srcm} J$ & Excinuclease $A B C$ subunit $A$ & 825 & CmrX [Streptomyces griseus subsp. griseus] & $79 \%$ \\
\hline $\mathrm{srcmC}$ & NDP-hexose C-methyltransferase & 409 & CmmC [Streptomyces griseus subsp. griseus] & $78 \%$ \\
\hline srcmUIII & 4-ketoreductase & 335 & StaK [Streptomyces sp. TP-A0274] & $50 \%$ \\
\hline srcmGIII & glycosyltransferase & 400 & ChlC7 [Streptomyces antibioticus] & $41 \%$ \\
\hline $\operatorname{srcm} A$ & acetyltransferase & 422 & CmmA [Streptomyces griseus subsp. griseus] & $74 \%$ \\
\hline srcmGIV & glycosyltransferase & 391 & PyrC4 [Streptomyces rugosporus] & $41 \%$ \\
\hline srcmGll & glycosyltransferase & 391 & SsfS6 [Streptomyces sp. SF2575] & $38 \%$ \\
\hline srcmF & dTDP-4-keto-6-deoxy-D-glucose epimerase & 199 & dTDP-4-keto-6-deoxy-D-glucose epimerase [Streptomyces tsukubensis] & $78 \%$ \\
\hline srcmOll & oxygenase & 550 & DacO2 [Dactylosporangium sp. SC14051] & $57 \%$ \\
\hline sremTll & ketoreductase & 253 & RubJ [Streptomyces collinus] & $50 \%$ \\
\hline srcmQ & aromatase & 320 & DacK [Dactylosporangium sp. SC14051] & $45 \%$ \\
\hline srcmLII & acyl CoA ligase & 536 & SsfL2 [Streptomyces sp. SF2575] & $48 \%$ \\
\hline
\end{tabular}

that this gene encodes a PadR-like family transcriptional regulator, and is a putative repressor. To identify the role of $s r c m R I I$ in chromomycin biosynthesis, we attempted to inactivate srcmRII in S. roseiscleroticus using a double crossover homologous recombination approach (Fig. 4b). To this end, we constructed pZJ196 that is a derivative of pKC1139 and harbors the homologous arms flanking srcmRII. After the transformation of $S$. roseiscleroticus with this plasmid through conjugation and subsequent homologous recombination, an engineered strain SR2 was obtained. PCR analysis showed that a short version of srcmRII was amplified from the mutant (Fig. 4c), confirming that this gene was successfully disrupted. This engineered strain was cultured onto R5 agar medium, with wild type $S$. roseiscleroticus as control. HPLC and ESI-MS analyses revealed that the wild type strain did not produce chromomycins in R5 agar, 


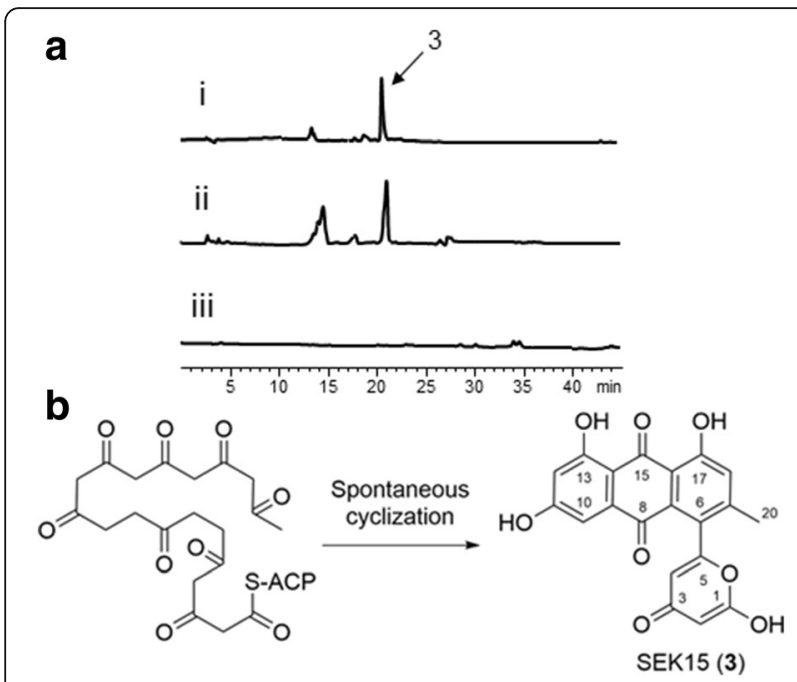

Fig. 3 Heterologous expression of SrcmPKS in S. lividans K4-114. a HPLC analysis of the extracts of S. lividans K4-114 harboring pSUN6 (i), pTZ3 (ii) and empty pRM5 vector (iii) respectively. Compounds were detected at $420 \mathrm{~nm}$. b Formation of SEK15b (3) through spontaneous cyclization of the nascent decaketide chain

while the engineered strain SR2 produced two new main products (traces i and ii, Fig. 4a).

The two main products $\mathbf{1}$ and $\mathbf{2}$ were purified and obtained as yellow powder. They were identified as chromomycins $A_{3}$ and $A_{2}$, respectively, based on spectroscopic analyses. Their molecular weights were determined to be $1210\left([\mathrm{M}-\mathrm{H}]^{-} \mathrm{m} / z\right.$ 1209.3, $[\mathrm{M}+\mathrm{Na}]^{+}$ $m / z 1233.4)$ and $1182\left([\mathrm{M}-\mathrm{H}]^{-} m / z\right.$ 1181.3, $[\mathrm{M}+\mathrm{Na}]^{+}$ $m / z$ 1205.4), respectively, based on the ESI-MS spectra (Additional file 1: Figure S1b-c). Their UV absorptions (Additional file 1: Figure S1b-c) were same as the previously reported [4]. The structures were further confirmed by comparing the ${ }^{1} \mathrm{H}$ NMR data (Additional file 1: Table S2) with those in the literature [21, 22]. In order to calculate the production of $\mathbf{1}$ and $\mathbf{2}$, we established a standard curve for the relationship between the amounts of each pure compound $(\mu \mathrm{g})$ and the peak areas $(420 \mathrm{~nm})$. Based on the standard curve, the titers of 1 and 2 in strain SR2 in R5 agar were determined to be $49.3 \pm 4.3$ and $53.3 \pm 3.6 \mathrm{mg} / \mathrm{L}$, respectively.

\section{Overexpression of SrcmRI in S. roseiscleroticus}

Besides srcmRII, the other regulatory gene srcmRI is a putative SARP, which was predicted to be the activator of the chromomycin biosynthetic pathway. To find out the role of srcmRI in the biosynthesis of chromomycins, we attempted to overexpress the srcmRI gene in $S$. roseiscleroticus. To this end, we constructed a pSET152-derived plasmid pZJ183 that harbors srcmRI under the strong constitutive promoter ermP. After the transformation of S. roseiscleroticus with pZJ183, we obtained the engineered strain SR1. The culture of

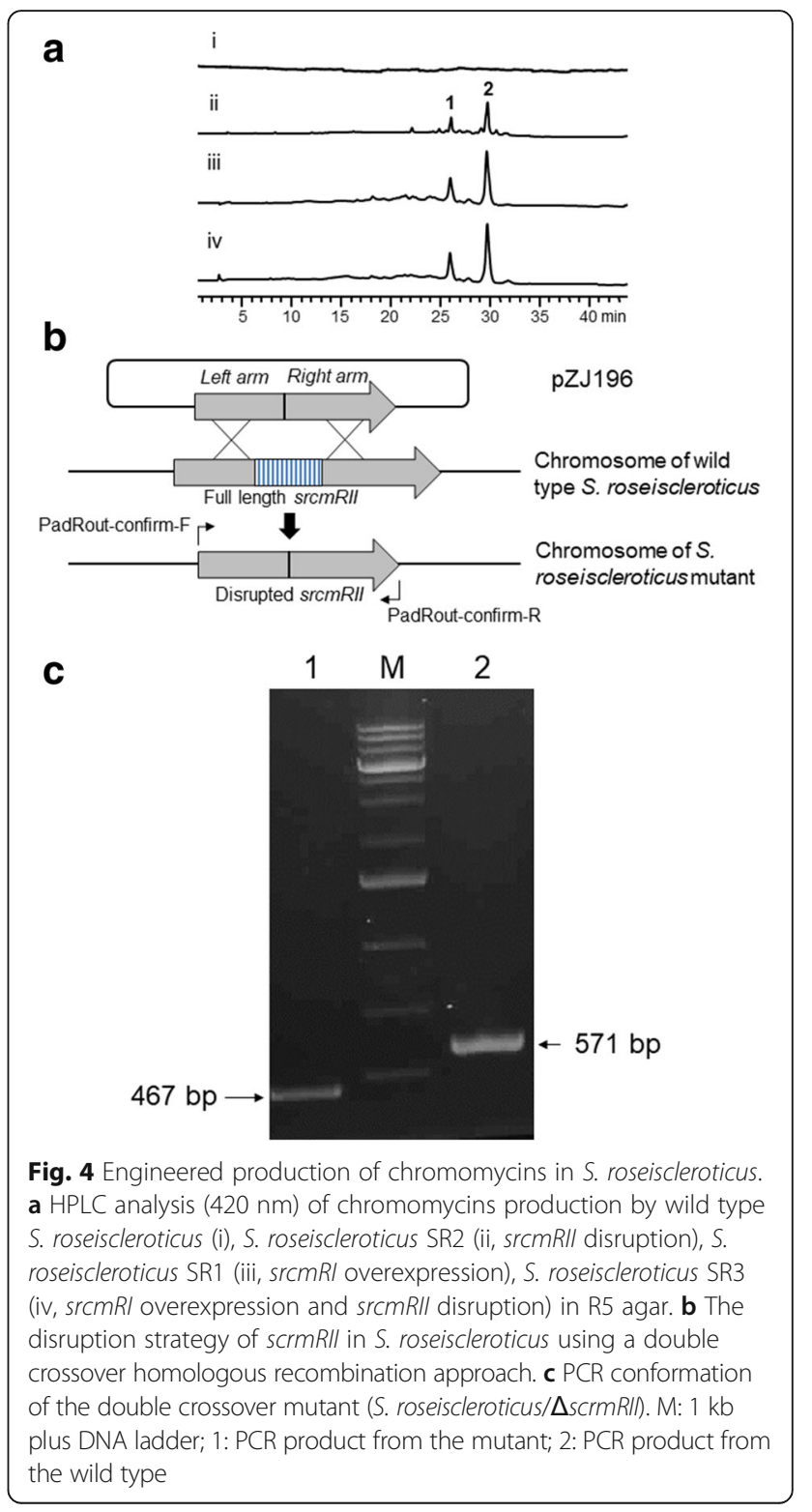

this strain in R5 agar was extracted and analyzed as described before. As shown in trace iii of Fig. 4a, compared to the wild type control (trace i, Fig. 4a), strain SR1 in R5 agar also produced $\mathbf{1}$ and 2 . The average titers of $\mathbf{1}$ and $\mathbf{2}$ were $8.9 \pm 1.2$ and $13.2 \pm$ $1.6 \mathrm{mg} / \mathrm{L}$, respectively.

\section{Optimization of the production of 1 and 2 in engineered S. roseiscleroticus}

According to our results, either inactivation of $s r c m R I I$ or overexpression of the srcmRI gene in S. roseiscleroticus "turned on" the chromomycin biosynthetic pathway, and led to the production of $\mathbf{1}$ and $\mathbf{2}$ at different titers. In order to further improve the production of $\mathbf{1}$ and $\mathbf{2}$, we tried overexpressing srcmRI in strain SR2, in which 
srcmRII was disrupted. The double crossover strain SR2 was used as host, and transformed with pZJ183 to get strain SR3, which has both disrupted srcmRII and overexpressed $\operatorname{srcmRI}$.

We cultured the engineered strain SR3 in R5 agar supplemented with apramycin, as described above, and the culture was extracted and analyzed. As shown in trace iv of Fig. 4a, strain SR3 also produced $\mathbf{1}$ and 2 . The titers of 1 and 2 were $69.4 \pm 7.6$ and $81.7 \pm 7.2 \mathrm{mg} / \mathrm{L}$, respectively. Compared to SR2 strain under the same culture conditions, the SR3 strain produced approximately $48 \%$ more chromomycins (1 and 2). Strain SR3 produced approximately 7.5 folds more than SR1 did.

Since malonyl-CoA is the substrate for assembly of the polyketide aglycon, more sugar in the medium might provide more carbon substrate for chromomycin biosynthesis. Additionally, sugar moieties are also present in the side chains of chromomycins. In order to provide more sugar in the medium, we attempted to use the yeast extract-malt extract (YEME) medium (containing $1 \%$ glucose and $17 \%$ sucrose) for chromomycins production. Three engineered strains SR1, SR2 and SR3 were cultured on YEME solid medium for 7 days. As shown in Fig. 5a, the production of $\mathbf{1}$ and $\mathbf{2}$ was significantly increased in the YEME culture. Compared to the cultures in $\mathrm{R} 5$ agar, the production (1 plus $\mathbf{2}$ ) in YEME was much higher, with a $58.3 \%$ increase for SR1, $98.9 \%$ increase for SR2, and 95.8\% increase for SR3. The titers of $\mathbf{1}$ and $\mathbf{2}$ in SR3 were $138.5 \pm 15.6$ and $157.3 \pm 13.8 \mathrm{mg} / \mathrm{L}$, respectively.

These results further confirmed that SR3, with srcmRI overexpression and srcmRII disruption, has higher production of chromomycins than the other two strains. Considering further application of these engineered strains in industry, we cultured SR3 in YEME liquid medium for time course analysis. The HPLC results (Fig. 5b) showed that the titer has been increasing in the first 3 days, and then reached a stable level. The highest titer of $\mathbf{1}$ was $145.1 \pm$ $15.3 \mathrm{mg} / \mathrm{L}$, and that of 2 was $158.3 \pm 15.4 \mathrm{mg} / \mathrm{L}$. This engineered strain provides a great starting strain for efficient production of these two products in the pharmaceutical industry.

\section{Discussion}

The genomic DNA of S. reseiscleroticus was sequenced by our group, and a gene cluster of 36 genes were predicted to be involved in the biosynthesis of chromomycins. The proposed biosynthetic pathway of $\mathbf{1}$ and $\mathbf{2}$ is shown in Fig. 2b. The minimal PKS consisting of SrcmP (ketosynthase), SrcmK (polyketide chain length factor) and SrcmS (acyl carrier protein) was proposed to synthesize a decaketide backbone. Heterologous expression of SrcmP, K and $\mathrm{S}$ in S. lividans yielded 3, a

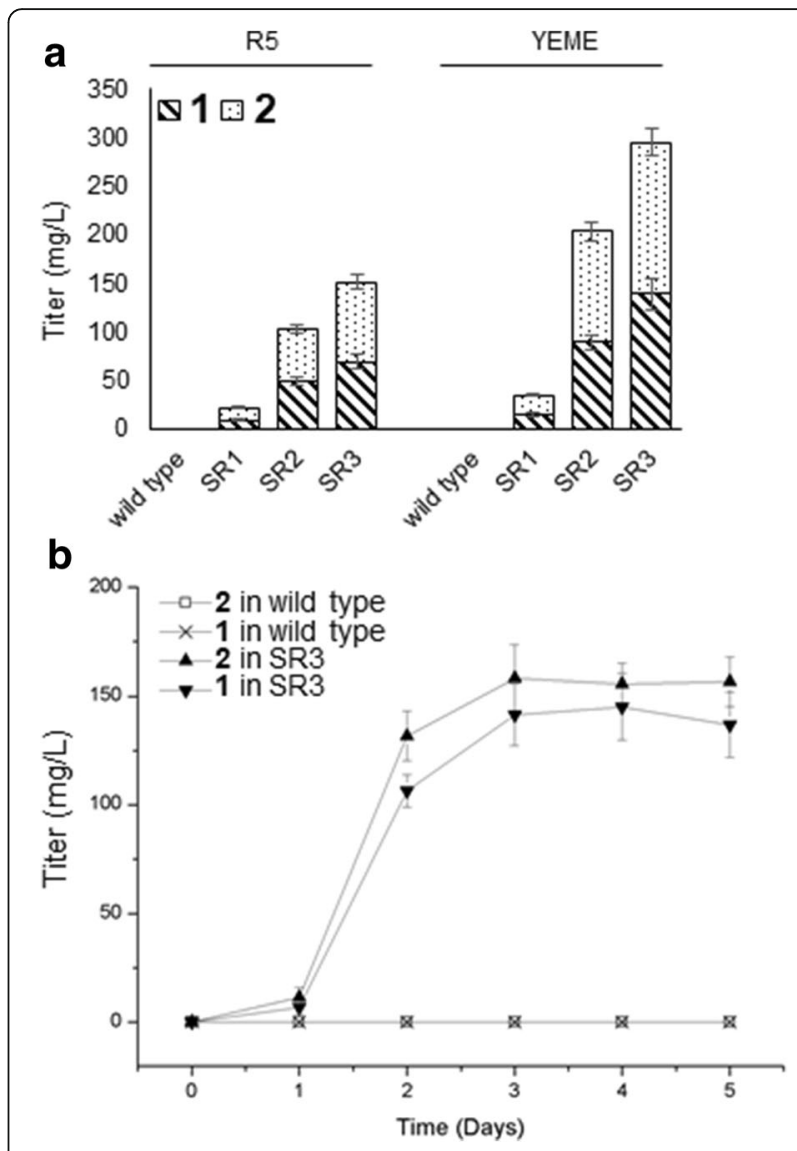

Fig. 5 Optimization of chromomycins production. a Production of $\mathbf{1}$ and $\mathbf{2}$ in two different solid media. $\mathbf{b}$ Time course analysis of chromomycins production of in liquid YEME medium. Error bars represent the standard deviation from three replicates

spontaneously cyclized decaketide product, confirming that the srcm minimal PKS synthesizes a decaketide chain for chromomycin biosynthesis. The nascent decaketide backbone will be cyclized with the participation of the aromatase SrcmQ to form the first ring, which will be further folded and modified by the cyclases (SrcmX and SrcmY) and ketoreductases (SrcmTI and SrcmTII) to form a four-ring structure. Two hydroxyl groups will be introduced by the oxygenases SrcmOI and SrcmOII to form 4-demethyl-premithramycinone. The methylation on 1'-hydroxy group is proposed to be catalyzed by SrcmMI, a putative O-methyltransferase, leading to the synthesis of premithramycinone. After the synthesis of aglycon part, several steps of glycosylation will occur. Additional structural modifications by other tailoring enzymes and the four glycosyltransferases (SrcmGI-IV) afford the ultimate structure of $\mathbf{1}$ and $\mathbf{2}$ (Fig. 2b). The type II polyketide biosynthetic gene cluster we found in S. reseiscleroticus is fully consistent with the previously reported chromomycin biosynthetic gene cluster [1]. While we confirmed the functions of the 
minimal PKS in chromomycin biosynthesis in the present work, the remaining biosynthetic enzymes can be further characterized through heterologous expression or gene knockout in the future work. Understanding of these enzymes and corresponding biosynthetic steps may further facilitate rational engineering of the chromomycin biosynthetic pathway for enhanced production of these pharmaceutically important molecules or generation of new chromomycin analogs.

The srcm gene cluster contains two putative regulatory genes, respectively encoding a SARP-type activator SrcmRI and a PadR-like repressor SrcmRII which was predicted to be a transcriptional inhibitor in chromomycin resistance/biosynthesis in the producing microorganism $[1,15]$. It was previously reported that disruption of PadR-like repressor CmmRII increased the production of 1 by approximately 70\% [15]. Overexpression of the SARP-type activator gene asm18 in Actinosynnema pretiosum increased the titer of $\mathrm{N}$-demethyl-4, 5-desepoxy-maytansinol to $50 \mathrm{mg} / \mathrm{L}$, which was increased by 4.7 -fold compared to the wild type strain [23]. Similarly, mtmR from Streptomyces argillaceus was identified as a SARP regulatory gene. Replacement of this gene with an antibiotic resistance gene abolished the production of mithramycin, while overexpression of $m t m R$ in $S$. argillaceus led to a 16 -fold increase in mithramycin production [18]. In S. roseiscleroticus, we identified two regulatory genes in the scrm biosynthetic gene cluster, and tried overexpression of SrcmRI and deletion of SrcmRII to enable and increase the production of chromomycins. Overexpression of SrcmRI turned the wild type to an efficient chromomycin producer (22.1 $\mathrm{mg} / \mathrm{L}$ of chromomycins). Disruption of the repressor SrcmRII showed an even more significant effect on chromomycins production $(102.6 \mathrm{mg} / \mathrm{L}$ of chromomycins). This phenomenon suggests that SrcmRII has high activity on the inhibition of the biosynthetic pathway, which also explains why the wild type does not produce chromomycins under lab conditions. The silent chromomycin biosynthetic pathway was activated by disrupting the PadR-like repressor SrcmRII or overexpressing the SARP-type activator SrcmRI in S. roseiscleroticus. It was also found that combination of SrcmRII disruption and SrcmRII overexpression showed a synergistic effect that further increased the production titers and yielded a more efficient producing strain of chromomycins. This work thus demonstrates a new method to improve the production of target products by simultaneously manipulating both the pathway activator and repressor.

\section{Conclusions}

In conclusion, we sequenced the genome of S. reseiscleroticus and discovered a type II polyketide biosynthetic gene cluster consisting of 36 open reading frames, which was predicted to be involved in chromomycin biosynthesis. Heterologous expression of the minimal PKS encoded by srcmPKS in S. lividans K4114 confirmed that these enzymes work collaboratively to synthesize the decaketide backbone of chromomycins. In the srcm gene cluster, two putative regulatory genes were identified, including $\operatorname{srcmRI}$ encoding a SARP-type activator and srcmRII encoding a PadR-like repressor. Overexpression of SrcmRI activated the chromomycin biosynthetic pathway and biosynthesized $\mathbf{1}$ and $\mathbf{2}$, whereas the disruption of SrcmRII increased the production much more than SrcmRI overexpression. In order to optimize the production of chromomycins, another engineered strain SR3 was constructed by both overexpressing SrcmRI and disrupting SrcmRII. Under the optimized culture conditions, 1 was produced at $145.1 \pm 15.3 \mathrm{mg} / \mathrm{L}$ and 2 at $158.3 \pm 15.4 \mathrm{mg} / \mathrm{L}$. Therefore, the inactivation of PadR-like repressor SrcmRII and overexpression of the SARP-type activator SrcmRI transformed S. roseiscleroticus from a non-producing strain into an efficient producer of $\mathbf{1}$ and $\mathbf{2}$. The engineered strain showed the promise of application in the pharmaceutical industry.

\section{Methods}

\section{General method}

Products were analyzed and purified on an Agilent 1200 HPLC instrument. ESI-MS spectra were obtained on an Agilent 6130 quadrupole LC-MS. NMR spectra were recorded on a JEOL NMR instrument $\left(300 \mathrm{MHz}\right.$ for ${ }^{1} \mathrm{H}$ NMR, $75 \mathrm{MHz}$ for ${ }^{13} \mathrm{C}$ NMR). NMR spectra were collected in $\mathrm{CDCl}_{3}$ for $\mathbf{1}$ and $\mathbf{2}$, and in DMSO- $d_{6}$ for $\mathbf{3}$. The chemical shift $(\delta)$ values are given in parts per million (ppm). The coupling constants $(J$ values) are reported in Hertz $(\mathrm{Hz})$.

\section{Strains, culture conditions, and plasmids}

E. coli DH5 $\alpha$ and ET12567 were grown in Luria-Bertani broth. S. roseiscleroticus (ATCC ${ }^{\circ}$ 53903) and S. lividans K4-114 [24] was cultured in ISP4 medium for seed culture preparation. R5 agar and YEME medium were used as the fermentation medium for chromomycins production, and TSBY salt medium for double crossover $[10,16]$. For conjugation, the Streptomyces strains were grown in TSB medium. R5 medium was used for genomic DNA isolation. Plasmid pRM5 [25] was used for heterologous gene expression in S. lividans K4-114. For gene manipulations in $S$. roseiscleroticus, plasmid pSET152 [26] was used for gene overexpression, and pKC1139 [26] for gene disruption. The concentrations of antibiotics used were $50 \mu \mathrm{g} / \mathrm{mL}$ for ampicillin, $50 \mu \mathrm{g} /$ $\mathrm{mL}$ for apramycin, $50 \mu \mathrm{g} / \mathrm{mL}$ for thiostrepton, and $50 \mu \mathrm{g} / \mathrm{mL}$ for nalidixic acid. 


\section{DNA manipulation and sequence analysis}

PCRs were performed using the Phusion DNA polymerase (Thermo Scientific, USA). S. roseiscleroticus and Streptomyces rimosus genomes were isolated by a standard procedure [27]. Conjugation was performed as described previously [28]. Homologous sequence database search and multiple sequence alignments were executed with BLASTP and ClustalX. The gene cluster was annotated with FramePlot [29] and was deposited in GenBank under accession number MG975976.

\section{Heterologous expression of minimal PKS in S. lividans K4-} 114

The minimal PKS genes srcmPKS were cloned from $S$. roseiscleroticus genome. The $s r c m P K$ genes were amplified using PCR with the primers KS-F-PacI and CLF-R-EcoRI-NheI (Table 2) and ligated into the pJET1.2 cloning vector (Thermo Fisher Scientific) to yield pZJ180 (Table 3). We amplified the $\operatorname{srcmS}$ gene with primers ACP-F-SpeI and ACP-R-NheI (Table 3) and ligated it into pJET1.2 to yield pZJ172 (Table 3). After sequencing, $\operatorname{srcm} S$ was excised from pZJ172 with SpeI and NheI and ligated into pZJ180 at the NheI site to yield pZJ178 (Table 3). The entire minimal PKS gene

Table 2 List of primers used in this study ${ }^{a}$

\begin{tabular}{|c|c|}
\hline Primers & Sequence \\
\hline KS-F-PaCl & $\begin{array}{l}\text { 5'-AATTAATTAAGGAGGAGCCCATATGAGAAGGCGC } \\
\text { GTCGTGGT-3' }\end{array}$ \\
\hline CLF-R-EcoRl-Nhel & $\begin{array}{l}\text { 5'-AAGAATTCGCTAGCCTACGGTCCCGACAGCGC } \\
\text { CA-3' }\end{array}$ \\
\hline OxyA-5-Pacl & $\begin{array}{l}\text { 5'-AATTAATTAAGGAGGAGCCCATATGTCCAAGATC } \\
\text { CATGACGC-3' }\end{array}$ \\
\hline OxyB-3-Nhel & 5'-AAGCTAGCTCAGTCCCGGCCGCTGACCA-3' \\
\hline OxyC-5-Spel & $\begin{array}{l}\text { 5'-AAACTAGTGGAGGAGCCCATATGACCCTGCTC } \\
\text { ACCCTCTC-3' }\end{array}$ \\
\hline OxyC-3-Nhel & 5'-AAGCTAGCTCACTTGTCCCGCGCGGCGC-3' \\
\hline ACP-F-Spel & $\begin{array}{l}\text { 5'-AAACTAGTGGAGGAGCCCATATGATGACGGTG } \\
\text { GACGATCT-3' }\end{array}$ \\
\hline ACP-R-Nhel & 5'- AAGCTAGCTCAGCCCACCCCGATGCCGT-3' \\
\hline $\begin{array}{l}\text { 14-2-SARP-F-Xbal- } \\
\text { Ndel }\end{array}$ & $\begin{array}{l}\text { 5'- AATCTAGAGGAGGAGCCCATATGAGCTCCGAC } \\
\text { AGCG } \overline{A C T G C A}-3^{\prime}\end{array}$ \\
\hline 14-2-SARP-R-Xbal & 5'- AATCTAGATCAGCCGGCCCGGCGCTGCC-3' \\
\hline $\begin{array}{l}\text { PadRout-left-F- } \\
\text { EcoRl }\end{array}$ & $\begin{array}{l}\text { 5'- AAA GAATTC TACTCGCCGGAGTGCGCACGGTCG } \\
\text { AA-3' }\end{array}$ \\
\hline $\begin{array}{l}\text { PadRout-left-R- } \\
\text { Xbal }\end{array}$ & 5'- AAA TCTAGA CTTCTTCGCGCAGCTTGCGCAGCT-3' \\
\hline $\begin{array}{l}\text { PadRout-right-F- } \\
\text { Xbal }\end{array}$ & 5'- AAA TCTAGA CGTACCGCTGTCGCCGCGAT-3' \\
\hline $\begin{array}{l}\text { PadRout-right-R- } \\
\text { Hindlll }\end{array}$ & 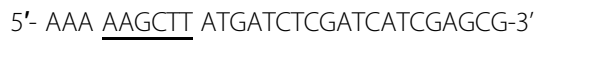 \\
\hline PadRout-confirm-F & 5'-AACATATGGCTCTGGGCACGCTGCA-3' \\
\hline PadRout-confirm-R & 5'-AAGAATTCTCACTGCGGGTCCTCCTGCG-3' \\
\hline
\end{tabular}

${ }^{\mathrm{a}}$ The restriction sites are underlined
Table 3 List of strains and plasmids used in this work

\begin{tabular}{|c|c|c|}
\hline Strains & Genotype & Source \\
\hline S. roseiscleroticus & Wild type & ATCC \\
\hline $\begin{array}{l}\text { S. lividans } \\
\text { K4-114 }\end{array}$ & $\begin{array}{l}\text { pro-2 str-6SLP2 - SLP3 - act::ermE } \\
\text { Streptomyces }\end{array}$ & [24] \\
\hline SR1 & $\begin{array}{l}\text { S. roseiscleroticus/pZJ183 (srcmRl } \\
\text { overexpression) }\end{array}$ & This Work \\
\hline SR2 & $\begin{array}{l}\text { S. roseiscleroticus/pZJ196 } \\
\text { (srcmR/l disruption) }\end{array}$ & This Work \\
\hline SR3 & $\begin{array}{l}\text { S. roseiscleroticus/pZJ183 + pZJ196 } \\
\text { (srcmRI overexpression and srcmRII } \\
\text { disruption) }\end{array}$ & This Work \\
\hline KS1 & S. lividans K4-114/pSUN6 & This Work \\
\hline Plasmids & Description & Source \\
\hline pZJ172 & srcms in pJET1.2 & This Work \\
\hline pZJ178 & srcmPKS in pJET1.2 & This Work \\
\hline pZJ179 & srcmRI in pJET1.2 & This Work \\
\hline pZJ180 & remPK in pJET1.2 & This Work \\
\hline pZJ178 & srcmPKS in pJET1.2 & This Work \\
\hline pZJ179 & srcmRl in pJET1.2 & This Work \\
\hline pSUN6 & srcmPKS in pRM5 & This Work \\
\hline pTZ3 & OxyABC in $p R M 5$ & This Work \\
\hline pZJ183 & srcmRl in PSET152 & This Work \\
\hline pZJ191 & srcmRIl knockout left arm in pJET1.2 & This Work \\
\hline pZJ192 & srcmRll knockout right arm in pJET1.2 & This Work \\
\hline pZJ195 & srcmRll knockout left arm in pKC1139 & This Work \\
\hline pZJ196 & $\begin{array}{l}\text { srcmRIl knockout left arm-right arm } \\
\text { in pKC1139 }\end{array}$ & This Work \\
\hline
\end{tabular}

cassette was then cut from pZJ178 using PacI and NheI and ligated into pRM5 (with a thiostrepton resistance gene) to yield pSUN6 (Table 3). Introduction of pSUN6 into the host strain S. lividans K4-114 via protoplast transformation afforded strain KS1 (Table 3). Using the same cloning strategy, the $\operatorname{oxy} A B$ genes were amplified from the genomic DNA of $S$. rimosus with primers OxyA-5-PacI and OxyB-3-NheI, and the $o x y C$ gene was amplified using PCR with primers OxyC-5-SpeI and OxyC-3-NheI (Table 2). These genes were combined in pJET1.2 and ligated into pRM5 between PacI and NheI to yield pTZ3 (Table 3).

\section{Overexpression of srcmRI in S. roseiscleroticus}

This srcmRI gene was PCR amplified with primers 142-SARP-F-XbaI-NdeI and 14-2-SARP-R-XbaI (Table 2) and ligated into pJET1.2 to yield pZJ179 (Table 3). After sequencing, the gene was excised from pZJ179 and ligated into pSET152 (with an apramycin resistance gene) at the $X b a I$ site under the ermE promoter to yield pZJ183. The $N d e I$ site included in the forward primer was used to determine the correct direction. Introduction of pZJ183 (Table 3 ) into wild type $S$. roseiscleroticus by protoplast transformation afforded the recombinant strain SR1 
(Table 3), in which the expression of $s r c m R I$ is under the control of the constitutive ermE promoter [27].

\section{Gene disruption of srcmRII in S. roseiscleroticus}

The srcmRII gene was inactivated by gene replacement to construct a $\Delta s r c m R I I$ mutant. The homologous arms were amplified with two sets of primers. PadRout-left-F-EcoRI and PadRout-left-R-XbaI (Table 2) were used to amplify the left arm, which was ligated into pJET1.2 to yield pZJ191 (Table 3). The fragment was subsequently ligated into pKC1139 at EcoRI/XbaI sites to yield pZJ195 (Table 3). PadRout-right-F-XbaI and PadRout-right-R-HindIII (Table 2) were used to amplify the right arm, which was cloned into pJET1.2 to yield pZJ192 (Table 3). The right arm was excised from pZJ192 with XbaI and HindIII and subsequently ligated into pZJ195 between the same sites to yield pZJ196 (Table 3). After introduction of pZJ196 into wild type S. roseiscleroticus strain via E. coli-S. roseiscleroticus conjugation, transformants were cultured at $37^{\circ} \mathrm{C}$ to screen for an apramycin-resistant phenotype to identify single crossover mutants. The single crossover mutants were cultured on blank TSBY salt plates for several generations, and then transferred onto the TSBY salt plates with or without apramycin. After 7 days' culture, the TSBY plates were screened for the double crossover colonies, which should have lost the apramycin-resistant phenotype. The strains that grew on blank TSBY salt plates, but did not grow with apramycin, were selected as double crossover candidates. The double crossover candidates were further confirmed by PCR with primers PadRout-confirm-F and PadRout-confirm-R (Table 2). The PCR product was also sequenced for further confirmation. After the double crossover strain was confirmed, it was named as SR2. pZJ183 was transferred into the SR2 strain via conjugation to yield SR3, which had both srcmRII disruption and srcmRI overexpression.

\section{Detection and purification of chromomycin $A_{3}$ (1), chromomycin $A_{2}$ (2) and SEK15b (3)}

The engineered $S$. roseiscleroticus or S. lividans K4114 strains grown on solid media were extracted with the method described before [1]. The liquid culture broths of the S. roseiscleroticus or S. lividans K4-114 strains were first centrifuged at $3000 \times \mathrm{g}$ for $10 \mathrm{~min}$ to harvest the cells. The supernatants were extracted three times with an equal volume of ethyl acetate, and the pellet cells were extracted with methanol. The two extracts were combined and dried with rotavapor. The residues were dissolved in $10 \mathrm{~mL}$ of methanol for HPLC analysis. The products were analyzed on an Agilent 1200 HPLC instrument (Agilent eclipse plus $\mathrm{C}_{18}, 5 \mu \mathrm{m}, 4.6 \mathrm{~mm} \times 250 \mathrm{~mm}$ ) using a linear gradient of 10 to $90 \%$ acetonitrile-water (containing $0.1 \%$ trifluoroacetic acid) over $45 \mathrm{~min}$ at a flow rate of $1 \mathrm{~mL} / \mathrm{min}$.

To establish the standard curves, chromomycins were purified from $500 \mathrm{~mL}$ of culture of strain SR2 in R5 agar. The crude extract was fractionated using a silica gel-60 column, eluted with different ratios of methanol-chloroform $(0: 100,1: 99,2: 98,3: 97,5: 95$, $10: 90,15: 85, v / v$, each $250 \mathrm{~mL}$ ) to afford seven fractions. The fractions containing the target compounds were further separated by HPLC (Agilent Eclipse Plus $\mathrm{C}_{18}, 5 \mu \mathrm{m}, 4.6 \mathrm{~mm} \times 250 \mathrm{~mm}$ ), eluted with acetonitrile-water at a flow rate of $1 \mathrm{~mL} \mathrm{~min}^{-1}$. 2, found in fraction 2, was further purified using HPLC with an isocratic elution of $47 \%$ acetonitrile-water. The target peak at $15.4 \mathrm{~min}$ was collected to yield $22.3 \mathrm{mg}$ of $\mathbf{2}$ in pure form. 1 was found in fraction 3 and was purified by HPLC with an isocratic elution of $43 \%$ acetonitrile-water. The target peak at $13.6 \mathrm{~min}$ was collected to yield $10.5 \mathrm{mg}$ of $\mathbf{1}$ in pure form.

The pure compounds were dissolved in $1 \mathrm{~mL}$ of methanol:DMSO ( $v / \mathrm{v}$ 50:50), which were serially diluted to obtain different concentrations. For each concentration, $100 \mu \mathrm{L}$ of these samples were injected into the HPLC to obtain the peak areas to establish standard curves for 1 and 2. Standard curves based on the linear relationship between the amounts $(\mu \mathrm{g})$ and the peak areas $(420 \mathrm{~nm})$ were established for $\mathbf{1}(\mathrm{y}=114.38 \mathrm{x}$ 853.13, $\left.\mathrm{R}^{2}=0.9965\right)$ and $2\left(\mathrm{y}=44.436 \mathrm{x}+196.1, \mathrm{R}^{2}=\right.$ 0.9912) for quantification of their production titers in different engineered strains. 3 was extracted from $500 \mathrm{~mL}$ of liquid culture broth of strain KS1 with an equal volume of ethyl acetate for three times. After evaporation of the solvent, the crude extract was also fractionated on a silica gel-60 column using the same procedure as described above. 3 was observed in fraction 4. It was purified using HPLC by an isocratic elution with $37 \%$ acetonitrile-water. The target peak at $17.1 \mathrm{~min}$ was collected to yield $9.1 \mathrm{mg}$ of $\mathbf{3}$ in pure form.

\section{Chromomycins production in liquid and solid media}

For the time-course analysis, $10^{6} / \mathrm{mL}$ spores of wild type $S$. roseiscleroticus or the engineered strain SR3 were inoculated respectively into $500 \mathrm{~mL}$ of liquid YEME with apramycin in a 2 - $\mathrm{L}$ flask at $28{ }^{\circ} \mathrm{C}$. The fermentation broths were sampled $(10 \mathrm{~mL})$ at $0,1,2,3,4$ and 5 days and the products were analyzed by HPLC using the above-mentioned method. For production analysis in solid medium, $10^{6} / \mathrm{mL}$ spores of wild type $S$. roseiscleroticus, engineered strains SR1, SR2 and SR3 were inoculated respectively onto R5 agar or YEME solid medium, and incubated at $28{ }^{\circ} \mathrm{C}$ for 7 days. 


\section{Additional file}

Additional file 1: Figure S1. UV and ESI-MS spectra of SEK15b (3, a), chromomycin $\mathrm{A}_{3}(\mathbf{1}, \mathrm{b})$ and chromomycin $\mathrm{A}_{2}(\mathbf{2}, \mathrm{c})$. Table S1. The ${ }^{1} \mathrm{H}$ $(300 \mathrm{MHz})$ and ${ }^{13} \mathrm{C}$ NMR $(75 \mathrm{MHz})$ data for SEK15b (3) $\left(\right.$ DMSO- $_{6}, \delta$ in ppm, $J$ in $\mathrm{Hz}$ ). Table S2. The ${ }^{1} \mathrm{H}$ NMR $(300 \mathrm{MHz})$ data for chromomycins $A_{3}$ (1) and $A_{2}$ (2) $\left(C C D C l_{3}, \delta\right.$ in ppm, J in Hz). (DOCX 200 kb)

\section{Abbreviations}

Pad: Phenolic acid decarboxylase; PKS: Polyketide synthase; SARP: Streptomyces antibiotic regulatory protein; YEME: Yeast extract-malt extract

\section{Funding}

This work was supported by a Utah State University Seed Program to Advance Research Collaborations (SPARC) Grant, a Grant-In-Aid from the American Heart Association (16GRNT26430067), and the Hundred Talents Program of Hunan Province, China.

\section{Availability of data and materials}

The DNA sequence of the chromomycin biosynthetic gene cluster is available to the public in NCBI under accession number MG975976.

\section{Authors' contributions}

JZhan supervised this study. JZhan and DY conceived and designed the experiments. LS, JZeng, PW, WW and DY performed the experiments. All authors analyzed the data and wrote the manuscript. All authors read and approved the final manuscript.

\section{Ethics approval and consent to participate}

Not applicable.

\section{Competing interests}

The authors declare that they have no competing interests.

\section{Publisher's Note}

Springer Nature remains neutral with regard to jurisdictional claims in published maps and institutional affiliations.

\section{Author details}

'Department of Biological Engineering, Utah State University, 4105 Old Main Hill, Logan, UT 84322-4105, USA. ${ }^{2}$ TCM and Ethnomedicine Innovation \& Development Laboratory, School of Pharmacy, Hunan University of Chinese Medicine, Changsha, Hunan 410208, China. ${ }^{3}$ Hangzhou Viablife Biotech Co., Ltd., 1 Jingyi Road, Yuhang District, Hangzhou, Zhejiang 311113, China.

Received: 11 May 2018 Accepted: 30 May 2018

Published online: 07 June 2018

\section{References}

1. Menéndez N, Nur-e-Alam M, Brana AF, Rohr J, Salas JA, Méndez C. Biosynthesis of the antitumor chromomycin $A_{3}$ in Streptomyces griseus. analysis of the gene cluster and rational design of novel chromomycin analogs. Chem Biol. 2004;11(1):21-32.

2. Lombo F, Menendez N, Salas JA, Mendez C. The aureolic acid family of antitumor compounds: structure, mode of action, biosynthesis, and novel derivatives. Appl Microbiol Biotechnol. 2006:73(1):1-14.

3. Skarbek JD, Speedie MK. Antitumor antibiotics of the aureolic acid group: chromomycin $A^{3}$, mithramycin $A$, and olivomycin A. In Antitumor Compounds of Natural Origin. (A. Aszalos, ed.). Chem Biochemistry. BocaRaton: CRC Press; 1981:1:191-235.

4. Bianchi N, Osti F, Rutigliano C, Corradini FG, Borsetti E, Tomassetti M, Mischiati C, Feriotto G, Gambari R. The DNA-binding drugs mithramycin and chromomycin are powerful inducers of erythroid differentiation of human K562 cells. Br J Haematol. 1999;104(2):258-65.

5. Chatterjee S, Zaman K, Ryu H, Conforto A, Ratan RR. Sequence-selective DNA binding drugs mithramycin a and chromomycin $A_{3}$ are potent inhibitors of neuronal apoptosis induced by oxidative stress and DNA damage in cortical neurons. Ann Neurol. 2001;49(3):345-54.
6. Bianchi N, Rutigliano C, Passadore M, Tomassetti M, Pippo L, Mischiati C, Feriotto G, Gambari R. Targeting of the HIV-1 long terminal repeat with chromomycin potentiates the inhibitory effects of a triplex-forming oligonucleotide on Sp1-DNA interactions and in vitro transcription. Biochem J. 1997;326(3):919-27.

7. Newman DJ, Cragg GM. Natural products as sources of new drugs over the last 25 years. J Nat Prod. 2007;70(3):461-77.

8. Wietzorrek A, Bibb M. A novel family of proteins that regulates antibiotic production in streptomycetes appears to contain an OmpR-like DNAblinding fold. Mol Microbiol. 1997;25(6):1181-4.

9. Barthelmebs L, Lecomte B, Divies C, Cavin JF. Inducible metabolism of phenolic acids in Pediococcus pentosaceus is encoded by an autoregulated operon which involves a new class of negative transcriptional regulator. J Bacteriol. 2000;182(23):6724-31.

10. Gury J, Seraut H, Tran NP, Barthelmebs L, Weidmann S, Gervais P, Cavin JF Inactivation of PadR, the repressor of the phenolic acid stress response, by molecular interaction with Usp1, a universal stress protein from Lactobacillus plantarum, in Escherichia coli. Appl Environ Microbiol. 2009;75(16):5273-83.

11. Tran NP, Gury J, Dartois V, Nguyen TKC, Seraut H, Barthelmebs L, Gervais P, Cavin J-F. Phenolic acid-mediated regulation of the padC gene, encoding the phenolic acid decarboxylase of Bacillus subtilis. J Bacteriol. 2008;190(9): 3213-24.

12. Kovacikova G, Lin W, Skorupski K. The virulence activator AphA links quorum sensing to pathogenesis and physiology in vibrio cholerae by repressing the expression of a penicillin amidase gene on the small chromosome. J Bacteriol. 2003:185(16):4825-36.

13. Huillet $E$, Velge $P$, Vallaeys $T$, Pardon P. LadR, a new PadR-related transcriptional regulator from listeria monocytogenes, negatively regulates the expression of the multidrug efflux pump MdrL. FEMS Microbiol Lett. 2006:254(1):87-94.

14. Agustiandari H, Lubelski J, van den Berg van Saparoea HB, Kuipers OP, Driessen AJM. LmrR is a transcriptional repressor of expression of the multidrug ABC transporter LmrCD in Lactococcus lactis. J Bacteriol. 2008; 190(2):759-63.

15. Menéndez N, Brana AF, Salas JA, Méndez C. Involvement of a chromomycin $A B C$ transporter system in secretion of a deacetylated precursor during chromomycin biosynthesis. Microbiology. 2007;153(9):3061-70.

16. Ohkuma $H$, Naruse N, Nishiyama $Y$, Tsuno T, Hoshino $Y$, Sawada $Y$, Konishi $M$, Oki T. Sultriecin, a new antifungal and antitumor antibiotic from Streptomyces roseiscleroticus. Production, isolation, structure and biological activity. J Antibiot. 1992;45(8):1239-49.

17. Prado L, Fernandez E, Weissbach U, Blanco G, Quiros LM, Brana AF, Mendez C, Rohr J, Salas JA. Oxidative cleavage of premithramycin B is one of the last steps in the biosynthesis of the antitumor drug mithramycin. Chem Biol. 1999:6(1):19-30.

18. Lombo F, Brana AF, Mendez C, Salas JA. The mithramycin gene cluster of Streptomyces argillaceus contains a positive regulatory gene and two repeated DNA sequences that are located at both ends of the cluster. J Bacteriol. 1999:181(2):642-7.

19. Zhang W, Ames BD, Tsai SC, Tang Y. Engineered biosynthesis of a novel amidated polyketide, using the malonamyl-specific initiation module from the oxytetracycline polyketide synthase. Appl Environ Microbiol. 2006;72(4): 2573-80.

20. McDaniel R, Ebert-Khosla S, Fu H, Hopwood DA, Khosla C. Engineered biosynthesis of novel polyketides: influence of a downstream enzyme on the catalytic specificity of a minimal aromatic polyketide synthase. Proc Natl Acad Sci U S A. 1994;91(24):11542-6.

21. Rohr J, Mendez C, Salas JA. The biosynthesis of aureolic acid group antibiotics. Bioorg Chem. 1999;27(1):41-54.

22. Miyamoto M, Kawamatsu Y, Kawashima K, Shinohara M, Nakanishi K. Full structures of three chromomycins, $A_{2}, A_{3}$, and A4. Tetrahedron Lett. 1966;(6):545-52.

23. Li S, Lu C, Chang X, Shen Y. Constitutive overexpression of asm 18 increases the production and diversity of maytansinoids in Actinosynnema pretiosum. Appl Microbiol Biotechnol. 2016;100(6):2641-9.

24. Ziermann R, Betlach MC. Recombinant polyketide synthesis in Streptomyces: engineering of improved host strains. BioTechniques. 1999;26(1):106-10.

25. McDaniel R, Ebert-Khosla S, Hopwood DA, Khosla C. Engineered biosynthesis of novel polyketides. Science. 1993:262(5139):1546-50.

26. Bierman M, Logan R, O'Brien K, Seno ET, Rao RN, Schoner BE. Plasmid cloning vectors for the conjugal transfer of DNA from Escherichia coli to Streptomyces spp. Gene. 1992;116(1):43-9. 
27. Kieser T, Bibb MJ, Buttner MJ, Chater KF, Hopwood DA. Practical Streptomyces Genetics. Norwich: The John Innes Foundation; 2000

28. Shao L, Zi J, Zeng J, Zhan J. Identification of the herboxidiene biosynthetic gene cluster in Streptomyces chromofuscus ATCC 49982. Appl Environ Microbiol. 2012;78(6):2034-8.

29. Ishikawa J, Hotta K. FramePlot: a new implementation of the frame analysis for predicting protein-coding regions in bacterial DNA with a high $\mathrm{G}+\mathrm{C}$ content. FEMS Microbiol Lett. 1999:174(2):251-3.

Ready to submit your research? Choose BMC and benefit from:

- fast, convenient online submission

- thorough peer review by experienced researchers in your field

- rapid publication on acceptance

- support for research data, including large and complex data types

- gold Open Access which fosters wider collaboration and increased citations

- maximum visibility for your research: over $100 \mathrm{M}$ website views per year 